\title{
Recursos fisioterapêuticos utilizados no Pós-COVID 19: Uma revisão bibliográfica
}

\author{
Physiotherapeutic resources used in Post-COVID 19: A literature review \\ Recursos fisioterapéuticos utilizados en Post-COVID 19: Una revisión de la literatura
}

Recebido: 03/06/2021 | Revisado: 13/06/2021 | Aceito: 14/06/2021 | Publicado: 27/06/2021

\author{
Bruna Pereira Nagamine \\ ORCID: https://orcid.org/0000-0002-8799-2529 \\ Instituto Educacional Santa Catarina, Brasil \\ Faculdade Guaraí, Brasil \\ E-mail: brunapnagamine@gmail.com \\ Lécia Kristine Lourenço \\ ORCID: https://orcid.org/0000-0002-0531-7221 \\ Instituto Educacional Santa Catarina, Brasil \\ Faculdade Guaraí, Brasil \\ E-mail: leciakristine@yahoo.com.br \\ Camila Teixeira de Oliveira Penna Chaves \\ ORCID: https://orcid.org/0000-0002-3008-2591 \\ Instituto Educacional Santa Catarina, Brasil \\ Faculdade Guaraí, Brasil \\ E-mail: camysto@hotmail.com
}

\begin{abstract}
Resumo
Pacientes pós-COVID-19 em sua grande maioria necessita de reabilitação fisioterapêutica durante e logo após a internação segundo a Organização Mundial da Saúde. E o programa de reabilitação fisioterapêutico possui uma ênfase e maior importância no sistema cardiorrespiratório. O objetivo desse artigo é demonstrar como os recursos fisioterapêuticos podem auxiliar na melhora desses pacientes Pós-Covid 19 independente do estado em que se encontra, seja ele leve, moderado ou grave, a partir das pesquisas realizadas nas plataformas digitais: Scielo, Pubmed, Associações Internacionais com os descritores coronavírus, fisiopatologia e tratamento, durante o período de 6 meses. Diante das pesquisas realizadas pode-se concluir que a reabilitação fisioterapêutica através de recursos como: thresold (aparelho com carga linear pressórica), RPPI (respiração com pressão positiva intermitente), ressuscitador manual e exercícios resistidos conseguem melhorar a capacidade cardiorrespiratória e promover uma maior tolerância ao recondicionamento dos pacientes Pós-Covid 19, consequentemente melhorando a qualidade de vida.
\end{abstract}

Palavras chave: Fisioterapia; Reabilitação; COVID-19.

\begin{abstract}
Abstrat
Most post-COVID-19 patients need physical therapy rehabilitation during and shortly after hospitalization according to the World Health Organization. And the physical therapy rehabilitation program has an emphasis and greater importance on the cardiorespiratory system. The aim of this article is to demonstrate how physical therapy resources can help improve these Post-Covid 19 patients, regardless of their status, whether mild, moderate or severe, based on research carried out on digital platforms: Scielo, Pubmed, Associações International with the descriptors coronavirus, pathophysiology and treatment, during a period of 6 months. Based on the researches carried out, it can be concluded that physical therapy rehabilitation through resources such as: thresold (linear pressure load device), RPPI (intermittent positive pressure breathing), manual resuscitator and resistance exercises can improve cardiorespiratory capacity and promote greater tolerance to the reconditioning of Post-Covid 19 patients, consequently improving their quality of life.
\end{abstract}

Keywords: Physiotherapy; Rehabilitation; COVID-19.

\section{Resumen}

La mayoría de los pacientes post-COVID-19 necesitan rehabilitación de físioterapia durante y poco después de la hospitalización según la Organización Mundial de la Salud, y el programa de rehabilitación de fisioterapia tiene un énfasis y una mayor importancia en el sistema cardiorrespiratorio. El propósito de este artículo es demostrar cómo los recursos de fisioterapia pueden ayudar a mejorar estos pacientes Post-Covid 19, independientemente de su estado, ya sea leve, moderado o severo, en base a investigaciones realizadas en plataformas digitales: Scielo, Pubmed, Associações International con los descriptores coronavirus, fisiopatología y tratamiento, durante un período de 6 meses. Con base en las investigaciones realizadas, se puede concluir que la rehabilitación fisioterapéutica a través de recursos como: thresold (dispositivo de carga de presión lineal), RPPI (respiración de presión positiva intermitente), 
resucitador manual y ejercicios de resistencia pueden mejorar la capacidad cardiorrespiratoria y promover una mayor tolerancia a el reacondicionamiento de los pacientes Post-Covid 19, mejorando consecuentemente su calidad de vida.

Palabras clave: Fisioterapia; Rehabilitación; COVID-19.

\section{Introdução}

O SARS-Cov-2 tem origem através de um vírus animal na qual pertencente à família dos Coronaviridae que incluem os vírus SARS-CoV e Síndrome Respiratória do Oriente Médio (MERS) e a infecção do SARS-CoV-2 provoca uma doença respiratória aguda denominada Coronavírus 2019 (COVID-19) e ao ser transmitido a sociedade, o covid19 provoca síndromes respiratórias graves como: a síndrome da dificuldade respiratória aguda (ARDS), síndrome respiratória aguda grave (SARS), síndrome respiratória do Oriente Médio(MERS) e atualmente, a COVID-19 (Fung et al., 2020; Lai et al., 2020; Mohd et al., 2016; Wernery et al., 2017; Wu et al., 2020; Ahmed et al., 2020 ; Cascella et al., 2020).

E o surgimento da atual pandemia está correlacionada com as síndromes de ARDS, SARS e MERS e as mutações relacionadas ao atual coronavírus (covid19) decorre de animais infectados tendo os morcegos como agente transmissor, onde o SARS-CoV-2 é um vírus de RNA com característica de fita única com um genoma de 30 quilobases que codificam múltiplas proteínas estruturais e é pertencente ao Coronaviridae família do betacoronavírus do gênero tipo 2 (Liang et. al. 2020). Além disso, o novo SARS-CoV-2 possui uma característica especifica ao se ligar a receptores da enzima conversora ACE2 que ao ligar nas células hospedeiras, os peptídeos do antígeno liberado pelo complexo de histocompatibilidade (MHC), citotóxicos (CTLs) e linfócitos $\mathrm{T}$ atuam na identificação de antígenos os reconhecendo como um agente estranho, dessa maneira reconhecendo o antígeno da SARS-CoV2 irá auxiliar a interpretar a patogênese da nova variante a COVID-19 (Prompetchara et al. 2020).

A transmissão do COVID-19 se dá através das gotículas respiratórias, partículas essas que estão presentes no ar ou quando um indivíduo entra em contato com um infectado (Chen, 2020).

O coronavírus em conjunto com a COVID-19 demonstra 75 - 80\% de similaridade com o coronavírus da SARS-CoV (síndrome respiratória aguda grave) estando interligado a tipo de coronavírus vindo do morcego (Riou e Althaus, 2020). Nos seres humanos o vírus se instala através dos receptores de ACE2 e em analises desenvolvidas demonstraram uma adaptação acelerada nas células hospedeiras (Malik et al., 2020).

A Covid-19 é uma patologia infectocontagiosa, ocasionada pelo vírus Sars-CoV-2, que possui como principal característica inflamações do sistema respiratório e os pacientes que desenvolveram comprometimentos graves podem desencadear síndromes pós cuidados intensivos, diminuição da capacidade cardiorrespiratória e limitações musculoesqueléticas. A fisioterapia vem ganhando destaque por atuar desde os cuidados hospitalares, até mesmo nas sequelas, através da reabilitação cardiopulmonar e musculoesquelética dos pacientes sequelados e diante das pesquisas o objetivo é demonstrar a eficiência de alguns recursos fisioterapêuticos que podem ser utilizado nos pacientes Pós-Cov19 (Silva,2020).

A reabilitação auxilia no recondicionamento do sistema cardiorrespiratório, nas limitações musculoesqueléticas, melhora da ventilação mecânica e capacidade funcional principalmente na realização das AVD’S, melhorando a fraqueza e fadiga (Gava; Picanço, 2007).

\section{Metodologia}

$\begin{array}{ccccccccc}\text { Este } \text { estudo } & \text { trata-se de uma revisão bibliográfica, descritiva e exploratória, de } \\ \text { abordagem qualitativa. } & \text { Tendo como base para sua discussão teórica, artigos científicos e }\end{array}$
demais produções intelectuais (Pereira et al., 2018).

O levantamento bibliográfico foi realizado nas bases de dados: sciELO, Pubmed, Associações Internacionais, com os descritores coronavírus, fisiopatologia e tratamento, no período de janeiro a junho de 2021. 
Foram encontrados 61 artigos, e apenas 27 foram para a pesquisa e os textos examinados foram criteriosamente avaliados por três autores independentes.

Foram considerados os critérios de artigos e produções intelectuais que demonstrasse eficiência como forma de tratamento de acordo com a sintomatologia que os pacientes estão apresentando no Pós-Covid19.

\section{Discussão e Resultados \\ Fisiopatologia da Doença}

A SARS-Cov2 se desenvolve de forma favorável mediante células epiteliais das vias aéreas humanas, usando células de enzima conversora de angiotensina 2 (ACE2) como receptora, fazendo com que a infeção se instale no momento que se tenha a infecção do trato respiratório inferior (Perlman, 2020).

O atual Covid19 possui a capacidade de se instalar no organismo humano de forma rápida devido a proteína Spike entrando nas células hospedeiras, que possui compatibilidade de 75,6 de aminoácidos idênticos do novo SARS-Cov2 e que passa por um processo de clivagem antes de penetrar nas células alvo (Zhang et al., 2020).

E a protease transmembranar, a serina 2 é a enzima que em humanos é codificada pelo gene TMPRSS2, que auxilia no desenvolvimento da homeostase e que é considerada um agente hospedeiro vital na propagação da COVID-19(D ' Amico et al., 2020).

O SARS-CoV-2 entra nas células por meio de difusão através da membrana retardando a regulação da ACE2, depois que consegue entrar se inicia o processo de replicação seguido de etapas de multiplicação, e devido os agentes lisossomo trópicos a infecção se torna mais grave e mais sensível desequilibrando o $\mathrm{pH}$ endossomal, nesse processo de infecção, os principais macrófagos e pneumócitos presentes nos pulmões são infectados (Docea et al., 2020; Silhol et al., 2020; Lan et al., 2020).

No processo de infecção proteases são liberadas, gerando espécies reativas de oxigênio (ROS) em todas as células hospedeira, e a liberação dos ROS é toxica para o sistema celular, no entanto, a partir da liberação dos ROS todo o mecanismo de defesa é ativado pelo sistema imunológico, e os primeiros agentes liberado pelo sistema imunológico são os neutrófilos pela infecção se estalar primeiro nos pulmões, seguido pelas demais células mieloides pertencente a resposta da imunidade inata (Nasi et al., 2020; Chen Y. et al., 2020).

Nos pacientes infectados pela COVID19 foi verificado reduzido o nível de lectina ligadora de manose (MBL), proteína associada a doenças auto imune e doenças infecciosas e os pacientes do COVID19 quando comparado com pacientes saudáveis e devido a esse fator disfunções imunológicas como as insuficiências respiratórias graves são características principais de pacientes com Covid-19 (Giamarellos-Bourboulis et al. 2020).

\section{Recursos fisioterapêuticos}

O programa de reabilitação fisioterapêutica pode ajudar na perda de peso e ganho de massa muscular, e a melhora do sistema cardiorrespiratório e os exercícios respiratórios são necessários para a reabilitação pulmonar com os objetivos de diminuir a fraqueza, dispneia, fadiga, melhorar a troca gasosa, aumentar a força dos músculos respiratórios (Machado, 2008).

A reabilitação pulmonar auxilia na suplementação de oxigênio, dispneia e na capacidade cardiorrespiratória. Pacientes que estram para reabilitação pós-covid 19 precisa adotar um estilo de vida mais saudável e ativo, para melhorar nos sintomas e sequelas. O descondicionamento físico e as alterações nas funções musculares periféricas aumentam as chances de fadiga e dispneia. A reabilitação pulmonar melhora a capacidade cardiorrespiratória e promove maior tolerância ao recondicionamento, melhorando a realização das AVDS'S (Gava; Picanço, 2007). 
Os exercícios resistidos tem se mostrado eficientes promovendo a melhora da força muscular, consequentemente, auxiliando na melhora da capacidade funcional, realização das AVD'S e prevenindo doenças adjacentes e o sedentarismo (Farias; Rodrigues, 2009).

Segundo o European Respiratory Journal o threshold (aparelho com carga linear pressórica é um recurso que traz benefícios por melhorar o quadro de dispneia, o trabalho respiratório, diminuindo o volume-minuto e o consumo de oxigênio e exerce uma função importante na reabilitação pulmonar e é um recurso para treinar os músculos respiratório que promove a reeducação funcional respiratória, aumentando a resistência diante de quadros de dispneia, aumentando a tolerância na realização das AVD'S e ao exercício e melhorando a força muscular inspiratória por propiciar carga inspiratória constante e sem alterações da pressão (Britto; Brant; Parreira, 2009).

O RPPI (respiração com pressão positiva intermitente) são exercícios respiratórios que se utiliza da pressão positiva para aumentar capacidade vital e o volume pulmonar, auxiliando na expansão pulmonar e tem como objetivos aumentar o volume corrente e a capacidade inspiratória. A expansão pulmonar que o RPPI realiza, aumenta a pressão intrapulmonar e o aparelho aumenta a pressão de abertura das vias aéreas, gerando diferença de pressão para ocorrer o fluxo inspiratório (Carvalho, 2009).

Ressuscitador manual é um recurso utilizado para ventilar manualmente e fornecer oxigênio através de ar comprimido e ventilação com pressão positiva aos pacientes para melhorar a expansão pulmonar e aqueles que necessitam de suporte ventilatório (AHA, 2005).

\section{Conclusão}

Embora não se saiba realmente as sequelas que o Covid-19 possa deixar, melhorar o condicionamento cardiorrespiratório e melhorar a força muscular dos músculos respiratórios é o primeiro passo para a reabilitação pós-covid além de serem uma das sequelas "padrões", que sabemos em maioria dos pacientes a patologia provoca o comprometimento pulmonar.

O tema abordado no presente artigo é de relevância cientifica, pois tem como objetivo auxiliar fisioterapeutas que trabalham com pós-covid, por se tratar de uma patologia nova e que ainda não se tem muitos estudos e pesquisas sobre recursos que podem ser utilizados na reabilitação fisioterapêutica.

\section{Referências}

Ahmed, S. F., Quadeer, A. A., \& McKay, M. R. (2020). Preliminary identification of potential vaccine targets for the COVID-19 coronavirus (SARS-CoV-2) based on SARS-CoV immunological studies. Viruses 12 (3), 254. doi: 10.3390/ v12030254

American Heart Association (AHA). (2005). Part 6: CPR Techniques and Devices. Circulation 2005;112:IV-47-50.

Britto, R. R.; brant, T. C. \& parreira, V. F. (2019). Recursos Manuais e Instrumentais em Fisioterapia Respiratória. Barueri, SP: Manole, 2009. Cad. Bras. Ter. Ocup., São Carlos, v. 27, n. 1, p. 27-34, 2019.

Carvalho, L.C. \& Pessoa, S.R. (2009). Epidemiologia da DPOC nos presentes aspectos nacionais. Rev. Pulmão Rj. Autorizações temáticas 2009. Vol. 1 Cascella, M., Rajnik, M., Cuomo, A., Dulebohn, S. C., and Di Napoli, R. (2020). Features, evaluation and treatment coronavirus (COVID-19) (StatPearls: StatPearls Publishing)

Chen, Y., Liu, Q., \& Guo, D. (2020). Emerging coronaviruses: genome structure, replication, and pathogenesis. J. Med. Virol. 92 (4), 418-423. doi: 10.1002/ jmv.25681

D’Amico, F., Baumgart, D. C., Danese, S., \& Peyrin-Biroulet, L. (2020). Diarrhea during COVID-19 infection: pathogenesis, epidemiology, prevention and management. Clin. Gastroenterol. Hepatol. 18, 1663-1672. doi: 10.1016/ j.cgh.2020.04.001

Docea, A. O., Tsatsakis, A., Albulescu, D., Cristea, O., Zlatian, O., Vinceti, M., et al. (2020). A new threat from an old enemy: Re emergence of coronavirus. Int. J. Mol. Med. 45 (6), 1631-1643. doi: 10.3892/ijmm.2020.4555

Farias I S R, \& Rodrigues T S. (2009). Exercício Resistido - Na saúde, na doença, no envelhecimento 2009. 
Fung, S. Y., Yuen, K. S., Ye, Z. W., Chan, C. P., \& Jin, D. Y. (2020). A tug-of-war between severe acute respiratory syndrome coronavirus 2 and host antiviral defence: lessons from other pathogenic viruses. Emerg. Microbes Infect. 9 (1), 558-570. doi: 10.1080/22221751.2020.1736644

Gava, Marcus V.; Picanço \& Patrícia S. A. (2006). Fisioterapia Pneumológica. São Paulo: Manole, 2007.

Giamarellos-Bourboulis, E. J., Netea, M. G., Rovina, N., Akinosoglou, K., Antoniadou, A., Antonakos, N., et al. (2020). Complex immune dysregulation in COVID-19 patients with severe respiratory failure. Cell Host Microbe. 16, 992-1000. doi: 10.1016/j.chom.2020.04.009

Lai, C. C., Shih, T. P., Ko, W. C., Tang, H. J., \& Hsueh, P. R. (2020). Severe acute respiratory syndrome coronavirus 2 (SARS-CoV-2) and corona virus disease2019 (COVID-19): the epidemic and the challenges. Int. J. Antimicrob. Agents, 105924. doi: 10.1016/j.ijantimicag.2020.105924

Liang, J., Mao, G., Yin, X., Ma, L., Liu, L., Bai, Y., et al. (2020). Identi fi cation and quanti fi cation of bacterial genomes carrying antibiotic resistance genes and virulence factor genes for aquatic microbiological risk assessment.

Machado M G R. (2008). Reabilitacao Pulmonar. In: Bases da Fisioterapia Respiratoria - Terapia Intensiva e Reabilitação. $1^{\text {a }}$ edição. Editora Guanabara Koogan, 2008.

Malik, Y. S., Sircar, S., Bhat, S., Vinodhkumar, O. R., Tiwari, R., Sah, R., et al. (2020). Emerging Coronavirus Disease (COVID-19), a pandemic public health emergency with animal linkages: Current status update. Preprints 2020030343. doi: 10.20944/preprints202003.0343.v1

Mohd, H. A., Al-Tawfiq, J. A., \& Memish, Z. A. (2016). Middle East respiratory syndrome coronavirus (MERS-CoV) origin and animal reservoir. Virol. J. 13 (1), 87. doi: 10.1186/s12985-016-0544-0

Nasi, A., McArdle, S., Gaudernack, G., Westman, G., Melief, C., Arens, R., et al. (2020). Proteasome and reactive oxygen species dysfunction as risk factors for SARS-CoV infection; consider N-acetylcystein as therapeutic intervention. Toxicol. Rep. 7, 768-771.

Pereira A.S. et al. (2018). Metodologia da pesquisa científica. [e-book]. Santa Maria. Ed. UAB/NTE/UFSM.

Perlman, S. (2020). Another decade, another coronavirus. N. Engl. J. Med. 382, 760-762. doi: 10.1056/NEJMe2001126

Prompetchara, E., Ketloy, C., \& Palaga, T. (2020). Immune responses in COVID-19 and potential vaccines: Lessons learned from SARS and MERS epidemic. Asian Pac. J. Allergy Immunol. 38 (1), 1-9.

Riou, J., \& Althaus, C. L. (2020). Pattern of early human-to-human transmission of Wuhan 2019 novel coronaviru-nCoV), December 2019 to January 2020. Eurosurveillance 25 (4), 2000058. doi: 10.2807/1560-7917.ES.2020.25.4.2000058

Silhol, F., Sarlon, G., Deharo, J. C., \& Vaïsse, B. (2020). Downregulation of ACE2 induces overstimulation of the renin-angiotensin system in COVID-19: should we block the renin-angiotensin system? Hypertension Res. 1-3. doi: 10.1038/ s41440-020-0476-3

Silva, R. M. V.; \& Sousa, A. V. C. (2020). Fase crônica da COVID-19: desafios do fisioterapeuta diante das disfunções musculoesqueléticas. Fisioter. Mov., Curitiba, v. 33, 2020

Wernery, U., Lau, S. K., \& Woo, P. C. (2017). Middle East respiratory syndrome (MERS) coronavirus and dromedaries. Vet. J. 220, 75-79. doi: 10.1016/ j.tvj1.2016.12.020

Wu, C., Liu, Y., Yang, Y., Zhang, P., Zhong, W., Wang, Y., et al. (2020b). Analysis of therapeutic targets for SARS-CoV-2 and discovery of potential drugs by computational methods. Acta Pharmaceutica Sinica B. doi: 10.1016/ j.apsb.2020.02.008

Zhang, H., Penninger, J. M., Li, Y., Zhong, N., \& Slutsky, A. S. (2020). Angiotensin-converting enzyme 2 (ACE2) as a SARS-CoV-2 receptor: molecular mechanisms and potential therapeutic target. Intensive Care Med. 1-5. doi: 10.1007/s00134-020-05985-9 